\title{
Pion decay of baryons in a flux-tube quark model
}

\author{
Fl. Stancu and P. Stassart \\ Institut de Physique B5, Sart Tilman, B-4000 Liege 1, Belgium \\ (Received 25 November 1987; revised manuscript received 26 February 1988)
}

\begin{abstract}
Pion decay widths of nonstrange-baryon resonances are calculated based on the ${ }^{3} P_{0}$ quark-paircreation model. The decay mechanism is consistent with the description of the nucleon and of the pion as having quark structure related to a flux-tube model motivated by QCD. The spectroscopic model has no adjustable parameter. The ${ }^{3} P_{0}$ model has one parameter which we fit to reproduce the $\Delta(1232)$ decay width. The results for 19 resonances are compared to experiment and to results obtained from the pseudoscalar-emission model. This sheds some light on the role of the finite size of the pion.
\end{abstract}

\section{INTRODUCTION}

Much progress has been made in understanding the hadron spectra through quark models inspired by QCD. Here we refer to flux-tube models abstracted from the strong-coupling Hamiltonian lattice formulation of QCD (Refs. 1 and 2). Such models can provide an adequate description of meson ${ }^{3,4}$ and baryon spectra ${ }^{3,5,6}$ and a more fundamental ground for the strong decay process via a flux-tube-breaking mechanism. The breaking mechanism supports in particular the ${ }^{3} P_{0}$ quark-pair-creation (QPC) model. ${ }^{7,8}$ Based on this model, in this paper we calculate the pion decay widths of nonstrange-baryon resonances. These resonances have been obtained ${ }^{5}$ by diagonalizing the hyperfine interaction (one-gluon exchange) in a basis provided by a semirelativistic Hamiltonian containing a relativistic kinetic-energy term and an adiabatic potential derived from a flux-tube quark model. ${ }^{3}$ We treat the pion as a finite-size particle and for consistency with the baryon description we assume its ground state to be described by the variational two-body correlation function of Ref. 3.

In Ref. 9 the pion decay widths were calculated on the basis of the same baryon resonance model but assuming a pseudoscalar-emission (PSE) model with a recoil term ${ }^{10,11}$ for the decay process. Accordingly the pion was treated as a pointlike particle. We compare the present results with those of Ref. 9 and with the local limit of the QPC model in order to study the effect of the structure of the pion.

In the next section we briefly describe the quark model for the $q \bar{q}$ and $q q q$ systems. In Sec. III the quark-paircreation model is reviewed. Results for the pion decay widths are given in Sec. IV and Sec. V is devoted to a discussion and summary.

\section{FLUX-TUBE MODEL FOR BARYONS AND MESONS}

An extensive presentation of the semirelativistic model used in this work to describe the baryon wave functions can be found in Refs. 5 and 9.

The three-quark Hamiltonian contains a relativistic kinetic-energy term and a potential-energy term which is the sum of three quark-quark potentials and of a threebody potential. The former consist of color Coulomb plus linear confinement terms. The latter is proportional to the string tension of the flux tube.

The parametrized ground-state wave function (which consists of two- and three-body correlation factors) has been obtained variationally in Ref. 1. Excited states up to two units of angular momentum or one unit of radial excitation are built orthogonally to the ground state. They are classified as the $56\left(0^{+}, 2^{+}\right), 56^{\prime}\left(0^{+}\right)$, $70\left(0^{+}, 1^{-}, 2^{+}\right)$, and $20\left(1^{+}\right) \mathrm{SU}(6)$ supermultiplets to be used with the spin-flavor states such as to give rise to totally symmetric states. Their degeneracy is broken by the hyperfine interaction ${ }^{12}$ containing spin-spin and tensor parts. Its singularities have been removed by including a form factor $e^{-(1 / 2) \Lambda^{2} q^{2}}$, where $\Lambda(\mathrm{fm})$ can be viewed as the "size of the quark," so as to mock up higher-order corrections beyond one-gluon exchange and neglected relativistic effects. The quark mass $m$ and size $\Lambda$ have been treated as parameters in Ref. 5. The values $m=324$ $\mathrm{MeV}, \Lambda=0.09 \mathrm{fm}$, hereafter called "set II," have been found to reproduce the mass of the ground-state nucleon and to give a good overall hadron spectrum. ${ }^{9}$

As discussed in the following section, the quark-paircreation model of strong interactions requires an explicit form for the radial part of the emitted meson. Calculations of decay widths of baryon resonances have been performed in Ref. 8 assuming a harmonic-oscillator wave function.

In this paper we deal with nonstrange particles only and both the meson and the baryon are consistently described by the same QCD-inspired quark model. ${ }^{1}$ Accordingly the meson is a $q \bar{q}$ system for which the fluxtube picture suggests the following Hamiltonian:

$$
H=\sum_{i=1,2}\left(p_{i}^{2}+m^{2}\right)^{1 / 2}-\frac{4}{3} \frac{\alpha_{s}}{\left|\mathbf{r}_{1}-\mathbf{r}_{2}\right|}+\sqrt{\sigma}\left|\mathbf{r}_{1}-\mathbf{r}_{2}\right|,
$$

where $m, r_{i}$, and $p_{i}$ are the quark (or antiquark) mass, position, and momentum, respectively. The values of the strong coupling constant $\alpha_{s}$ and string tension $\sqrt{\sigma}$ are taken as in Ref. 1: 


$$
\frac{4}{3} \frac{\alpha_{s}}{\hbar c}=0.5, \quad \sqrt{\sigma}=1 \mathrm{GeV} \mathrm{fm}^{-1}
$$

For consistency with the baryon wave function we choose for the lowest state of (2.1) the variational solution found in Ref. 1. Its explicit form reads

$f(r)=r^{-0.2} \exp \left\{-0.3965 r W(r)-2.1[1-W(r)] r^{1.5}\right\}$,

$W(r)=\frac{1+\exp (-0.15 / 0.05)}{1+\exp [(r-0.15) / 0.05]}$,

where $r$ is in fm. There is still some inconsistency with respect to the baryon wave function. Here for simplicity we neglect the one-gluon-exchange contributions to the meson wave function. In a more adequate treatment one should add the contribution of the hyperfine interaction or, better, consider the variational solution of Ref. 3 where the spin-spin, spin-orbit, and tensor correlations have been included. In a comparative study of $\pi$ and $\rho$ meson emission such a wave function would be necessary. In the above description $\pi$ and $\rho$ are different only in the flavor-spin space.

\section{QUARK-PAIR-CREATION MODEL FOR MESON EMISSION}

The decay model is based on the creation of a quarkantiquark pair with equal probability anywhere in the hadronic matter. The constant of the pair-creation process $\gamma$ is purely phenomenological. The quantum numbers of the $q \bar{q}$ pair are those of the vacuum, i.e., a ${ }^{3} P_{0}$ state. ${ }^{7,8}$ As shown in Fig. 1 the pair rearranges in a new baryon and an emitted meson together with the quarks of the decaying resonance, which are treated as spectators.

It has recently been shown ${ }^{4}$ that the ${ }^{3} P_{0}$ model is closely related to a flux-tube-breaking mechanism. It corresponds to the limiting case where the flux-tube wave function has an infinite extension. In this limit the amplitude for a ground-state flux tube (string) to break is equal at all points and independent of the transverse direction from the string. It can therefore be identified with the pair creation constant $\gamma$ of the ${ }^{3} \boldsymbol{P}_{0}$ model. Moreover, in the analysis of the meson decay it has been found that the flux-tube-breaking model with a finite extension for the wave function gives results very similar to the ${ }^{3} \boldsymbol{P}_{0}$ model. In keeping with this finding we use the ${ }^{3} \boldsymbol{P}_{0}$ model in this study of the baryon decay. This allows a substantial simplification of our calculations.

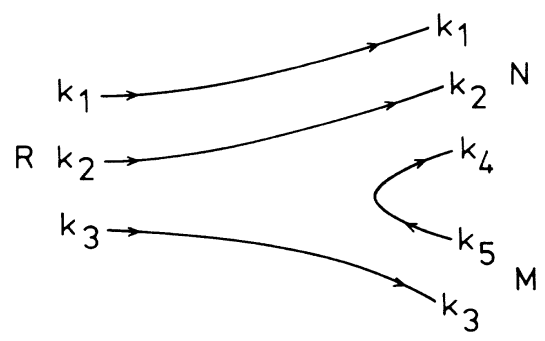

FIG. 1. The baryon-resonance decay into nucleon $N$ plus meson $M$.
According to Ref. 8 the decay amplitude for the process $R \rightarrow M N$, where $R$ is the baryon resonance, $M$ the meson, and $N$ the nucleon ground state in the rest frame of $R$, is given by

$$
\begin{aligned}
\langle N M|\hat{T}| R\rangle=\gamma \sum_{m} & \langle 11 m-m \mid 00\rangle\left\langle\Phi_{N} \Phi_{M} \mid \Phi_{R} \Phi_{\mathrm{vac}}^{-m}\right\rangle \\
& \times I_{m}(R ; N, M),
\end{aligned}
$$

where the $\Phi$ 's are the SU(6)-flavor-spin wave functions of the nucleon, meson, resonance, and of the vacuum and $I_{m}(R ; N, M)$ is the momentum-space integral:

$$
\begin{aligned}
I_{m}(R ; N, M)=\int & d \mathbf{k}_{1} d \mathbf{k}_{2} d \mathbf{k}_{3} d \mathbf{k}_{4} d \mathbf{k}_{5} \delta\left(\mathbf{k}_{1}+\mathbf{k}_{2}+\mathbf{k}_{3}\right) \\
& \times \delta\left(\mathbf{k}_{1}+\mathbf{k}_{2}+\mathbf{k}_{4}-\mathbf{k}_{N}\right) \delta\left(\mathbf{k}_{3}+\mathbf{k}_{5}-\mathbf{k}_{M}\right) \\
& \times \delta\left(\mathbf{k}_{4}+\mathbf{k}_{5}\right) Y_{1}^{m}\left(\mathbf{k}_{4}-\mathbf{k}_{5}\right) \psi_{R}\left(\mathbf{k}_{1}, \mathbf{k}_{2}, \mathbf{k}_{3}\right) \\
& \times \psi_{N}\left(\mathbf{k}_{1}, \mathbf{k}_{2}, \mathbf{k}_{4}\right) \psi_{M}\left(\mathbf{k}_{3}, \mathbf{k}_{5}\right)
\end{aligned}
$$

where the $\psi$ 's are the Fourier transforms of the spatial parts of $R, N$, and $M$ wave functions.

Using the Jacobi momentum coordinates

$$
\begin{aligned}
& \mathbf{k}_{\rho}=\left(\mathbf{k}_{1}-\mathbf{k}_{2}\right) / \sqrt{2}, \\
& \mathbf{k}_{\lambda}=\left(\mathbf{k}_{1}+\mathbf{k}_{2}-2 \mathbf{k}_{3}\right) / \sqrt{6}, \\
& \mathbf{K}=\frac{1}{\sqrt{3}}\left(\mathbf{k}_{1}+\mathbf{k}_{2}+\mathbf{k}_{3}\right),
\end{aligned}
$$

the integral $I_{m}$ becomes

$$
\begin{aligned}
I_{m}(R ; N, M)=\delta\left(\mathbf{k}_{M}+\mathbf{k}_{N}\right) \int & d \mathbf{k}_{\rho} d \mathbf{k}_{\lambda} \psi_{R}\left(\mathbf{k}_{\rho}, \mathbf{k}_{\lambda}\right) \\
& \times \psi_{N}\left(\mathbf{k}_{\rho}, \mathbf{k}_{\lambda}+\sqrt{2 / 3} \mathbf{k}_{M}\right) \\
& \times \psi_{M}\left(\mathbf{k}_{M}+2 \sqrt{2 / 3} \mathbf{k}_{\lambda}\right) \\
& \times Y_{1}^{m}\left(-2 \mathbf{k}_{M}-2 \sqrt{2 / 3} \mathbf{k}_{\lambda}\right) .
\end{aligned}
$$

It is convenient to rewrite $I_{m}$ in spatial coordinates, so that we can directly use the hadron wave functions described in Sec. II:

$$
\begin{aligned}
I_{m}(R ; N, M)=- & \left(\frac{3}{4 \pi}\right)^{/ 2} \frac{2^{3}}{(2 \pi)^{3 / 2}} \delta\left(\mathbf{k}_{M}+\mathbf{k}_{N}\right) \\
& \times \int \mathrm{d} \rho \mathrm{d} \lambda \mathrm{d} x \psi_{R}(\rho, \lambda+2 \sqrt{2 / 3} \mathbf{x}) \\
& \times \psi_{N}(\rho, \lambda) e^{i \mathbf{k}_{M}(\sqrt{2 / 3} \lambda+\mathbf{x})} \\
& \times \epsilon_{m}\left(\mathbf{k}_{M}+i \nabla_{x}\right) \psi_{M}(2 \mathbf{x})
\end{aligned}
$$

where $\rho, \lambda$ are the spatial Jacobi coordinates of the nucleon, $x$ is the relative coordinate of the outgoing pion, and $\epsilon_{m}$ is the spherical unit vector

$$
\epsilon_{ \pm 1}=\mp \frac{1}{\sqrt{2}}(1, \pm i, 0), \quad \epsilon_{0}=(0,0,1) \text {. }
$$


One can notice that the effect of the finite size of the meson is a change in the coordinate $\lambda$ of $\psi_{R}$ by an amount proportional to the relative distance $\mathrm{x}$ between $q$ and $\bar{q}$. This is a nonlocal effect specific to the QPC model. One can recover the pseudoscalar-emission (PSE) model by taking $\psi_{M} \sim \delta(2 \mathbf{x})$. To find the exact correspondence we first notice that there are six possible ways of rearranging the particles 1 to 5 into a nucleon and a pion, from which Fig. 1 is an example. The decay amplitude is then six times the expression (3.1). For $M=\pi^{0}$ one can reobtain the amplitudes given in Appen$\operatorname{dix} \mathbf{A}$ of Ref. 9 by making the replacement

$$
\begin{aligned}
& \sqrt{2} \psi_{\pi^{0}} Y_{10} \rightarrow \delta(2 \mathrm{x})\left(\mathcal{A}^{0}+\mathcal{D}^{0}\right), \\
& \psi_{\pi^{0}} Y_{1-1} \rightarrow \delta(2 \mathrm{x}) \mathcal{B}^{0} \\
& \psi_{\pi^{0}} Y_{11} \rightarrow-\delta(2 \mathrm{x}) \bigodot^{0}
\end{aligned}
$$

with $\mathcal{A}^{0}, \mathcal{B}^{0}, \mathcal{C}^{0}, \mathcal{D}^{0}$ defined in Ref. 9 and by using Eq. (3.11) of Ref. 9 relating the matrix elements of $\mathcal{B}^{0}$ and $\mathcal{C}^{0}$. Then the so-called two-parameter PSE model used in Ref. 9 has a one-to-one correspondence with the pointlike limit of the QPC model if one sets

$$
x=-y,
$$

where $x$ and $y$ are the two free parameters. This correspondence is useful in checking calculations based on the QPC model. The nine-dimensional integral (3.5) was solved numerically by a Monte Carlo method. Some details are given in the Appendix.

\section{RESULTS}

In the rest frame of a resonance of mass $m_{R}$ the decay width for the emission of a pion is given by

$$
\begin{aligned}
\Gamma_{N \pi}= & \frac{2}{\pi} \frac{|\langle N \pi|\hat{T}| R\rangle|^{2}}{2 J_{R}+1} \frac{k E_{\pi} E_{N}}{m_{R}} \\
& \times\left\langle I_{N} I_{\pi} I_{3 N} I_{3 \pi} \mid I_{R} I_{3 R}\right\rangle^{-2}
\end{aligned}
$$

where $J_{R}$ is the total angular momentum, $I$ the isospin, $I_{3}$ its projection, $k$ the pion momentum, $E_{\pi}$ its relativistic energy, and $E_{N}$ the relativistic recoil energy of the nucleon in its ground state.

The theoretical values of $\Gamma_{N \pi}^{1 / 2}$ calculated from expression (4.1) are presented in Table $I$. Column 5 is obtained by using the set II theoretical mass spectrum and the mixing angles ${ }^{9}$ both for the resonances and the nucleon ground state. Here we also indicate the signs of the associated amplitudes because they fix the signs of the helicity amplitudes for the radiative decay (see later). For the results labeled "hybrid" of column 4 the theoretical mass spectrum has been replaced by the experimental masses of the resonances under study. Column 3 shows results obtained $^{13}$ from the pointlike (local limit) of the QPC model. For three and four star resonances the experimental values are extracted from the baryon summary table of the Particle Data Group ${ }^{14}$ (PDG). For two star

\begin{tabular}{|c|c|c|c|c|c|c|}
\hline Resonance & Main component & $\begin{array}{c}\text { QPC } \\
\text { local limit } \\
\text { Set II }\end{array}$ & $\begin{array}{c}\text { QPC } \\
\text { hybrid }\end{array}$ & $\begin{array}{c}\text { QPC } \\
\text { Set II }\end{array}$ & Expt. & Status \\
\hline$P_{11}(1440)$ & ${ }^{2} N\left(56^{\prime}, 0^{+}\right) \frac{1}{2}^{+}$ & 4.9 & 21.7 & +20.8 & $10.9_{-3.2}^{+4.8}$ & $* * * *$ \\
\hline$D_{13}(1520)$ & ${ }^{2} N\left(70,1^{-}\right) \frac{3}{2}^{-}$ & 4.5 & 12.7 & +8.4 & $8.3_{-1.2}^{+0.9}$ & $* * * *$ \\
\hline$S_{11}(1535)$ & ${ }^{2} N\left(70,1^{-}\right) \frac{1}{2}^{-}$ & 18.3 & 7.3 & +6.3 & $8.0_{-2.1}^{+3.2}$ & $* * * *$ \\
\hline$S_{11}(1650)$ & ${ }^{4} N\left(70,1^{-}\right) \frac{1}{2}^{-}$ & 19.6 & 2.2 & +2.3 & $9.5_{-2.1}^{+1.9}$ & $* * * *$ \\
\hline$D_{15}(1675)$ & ${ }^{4} N\left(70,1^{-}\right) \frac{5}{2}^{-}$ & 3.1 & 8.3 & +5.6 & $7.6_{-1.1}^{+0.9}$ & $* * * *$ \\
\hline$F_{15}(1680)$ & ${ }^{2} N\left(56,2^{+}\right) \frac{5}{2}^{+}$ & 3.2 & 10.1 & +9.7 & $8.7_{-0.9}^{+0.8}$ & $* * * *$ \\
\hline$D_{13}(1700)$ & ${ }^{4} N\left(70,1^{-}\right) \frac{3}{2}^{-}$ & 2.3 & 5.4 & -4.1 & $3.2_{-1.3}^{+1.0}$ & $* * *$ \\
\hline$P_{11}(1710)$ & ${ }^{2} N\left(70,0^{+}\right) \frac{1}{2}^{+}$ & 3.5 & 3.3 & +1.8 & $4.0_{-1.0}^{+1.1}$ & $* * *$ \\
\hline$P_{13}(1720)$ & ${ }^{2} N\left(56,2^{+}\right) \frac{3}{2}+$ & 16.9 & 10.0 & -7.1 & $5.4_{-19}^{+1.7}$ & $* * * *$ \\
\hline$F_{17}(1990)$ & ${ }^{4} N\left(70,2^{+}\right) \frac{7}{2}^{+}$ & 0.6 & 2.5 & -1.8 & $4.2+1.99$ & $* *$ \\
\hline$F_{15}(2000)$ & ${ }^{4} N\left(70,2^{+}\right) \frac{5}{2}^{+}$ & 0.7 & 2.7 & -2.0 & $1.9_{-0.7}^{+0.7}$ & $* *$ \\
\hline$P_{33}(1232)$ & ${ }^{4} \Delta\left(56,0^{+}\right) \frac{3}{2}^{+}$ & 10.7 & 10.7 & +10.7 & $10.7_{-0.2}^{+0.2}$ & $* * * *$ \\
\hline$P_{33}(1600)$ & ${ }^{4} \Delta\left(56^{\prime}, 0^{+}\right) \frac{3}{2}{ }^{+}$ & 9.0 & 2.5 & -0.2 & $7.0_{-1.8}^{+2.4}$ & $* *$ \\
\hline$S_{31}(1620)$ & ${ }^{2} \Delta\left(70,1^{-}\right) \frac{1}{2}^{-}$ & 8.3 & 0.9 & -0.6 & $6.5_{-1.0}^{+1.0}$ & $* * * *$ \\
\hline$D_{33}(1700)$ & ${ }^{2} \Delta\left(70,1^{-}\right) \frac{3}{2}^{-}$ & 2.7 & 8.1 & -4.8 & $6.1_{-1.7}^{+1.6}$ & $* * * *$ \\
\hline$F_{35}(1905)$ & ${ }^{2} \Delta\left(70,2^{+}\right) \frac{5}{2}+$ & 1.2 & 3.6 & +3.1 & $5.5_{-2.0}^{+2.2}$ & $* * * *$ \\
\hline$P_{31}(1910)$ & ${ }^{2} \Delta\left(70,0^{+}\right) \frac{1}{2}^{+}$ & 7.8 & 1.0 & +0.7 & $6.6_{-1.1}^{+2.5}$ & $* * * *$ \\
\hline$P_{33}(1920)$ & ${ }^{4} \Delta\left(56,2^{+}\right) \frac{3}{2}^{+}$ & 1.7 & 1.6 & +2.0 & $6.6_{-1.3}^{+1.1}$ & $* * *$ \\
\hline$F_{37}(1950)$ & ${ }^{4} \Delta\left(56,2^{+}\right) \frac{7}{2}^{+}$ & 3.9 & 11.8 & -8.7 & $9.8_{-1.4}^{+2.6}$ & $* * * *$ \\
\hline
\end{tabular}
resonances an average of the data of Refs. 15 and 16 has been made, when both quoted in the full listings of PDG.

TABLE I. Square root of the decay width $\Gamma_{N \pi}^{1 / 2}$ in $\mathrm{MeV}^{1 / 2}$. Column 3: QPC, local limit (Ref. 13). Column 4: QPC, set-II mixing angles of Ref. 9 and experimental masses for the baryons, Eq. (2.3) for the pion. Column 5: same as column 4 but with theoretical masses of Ref. 9. Here the sign of the amplitudes is also indicated. Columns 6 and 7: data and status of resonances (Ref. 14). 
The constant $\gamma$ has been adjusted to reproduce the experimental value of $\Gamma_{N \pi}$ for $\Delta(1232)$. The values obtained for

$$
\chi^{2}=\sum_{N}\left(\frac{\sqrt{\Gamma_{\text {expt }}}-\sqrt{\Gamma_{\mathrm{th}}}}{\Delta \sqrt{\Gamma_{\text {expt }}}}\right)^{2},
$$

where $N$ is the number of resonances, are 206, 112, and 105 for the results in columns 3, 4, and 5, respectively. This shows that the replacement of the theoretical by the experimental masses slightly worsens the global fit. But the replacement of the point-particle pion by a finite-size pion required by the QPC model brings a substantial improvement decreasing the $\chi^{2}$ by a factor of 2 . We recall that the wave function (2.3) gives an rms radius of 0.29 fm for the pion. ${ }^{1}$ On the other hand, from set II we found a value of $0.37 \mathrm{fm}$ for the nucleon radius. The results of column 5 can also be compared to those named "all mix set II" in Ref. 9. The $\chi^{2}$ fit gave a value of 99 for 16 resonances there, i.e., comparable to 105 for 19 resonances here. But the present model has only one adjustable parameter while in Ref. 9 two free parameters have been used in the fit.

The difference is even more striking when one looks at the one-parameter PSE model, with a recoil term resulting from a standard nonrelativistic reduction. ${ }^{10}$ The $\chi^{2}$ over 16 resonances was found ${ }^{9}$ equal to 712 in that case. This comparison shows that the nonlocal character of the pion emission operator in the QPC model has a very important role as has already been stressed in an earlier study by Gavela et al. ${ }^{17}$ based on the harmonic-oscillator model. Also it suggests that the results of the twoparameter PSE model may be due to a mock-up of QPC nonlocal effects by the extra parameter.

Concerning the signs of the strong decay amplitudes the QPC model gives the same results as the PSE model ${ }^{9}$ except for the $P_{11}(1710)$ where the QPC model gives agreement with the experimental sign of the photodecay amplitudes calculated in Ref. 18 from the same quark model. This discussion is valid for the so-called "set II" model used in the present calculations.

In a detailed analysis we can see that in the present calculations most of the $\Gamma_{N \pi}^{1 / 2}$ lie within or near the experimental error interval. There are few striking exceptions though. These are the $P_{11}(1440), S_{11}(1650), P_{33}(1600)$, $S_{31}(1620), P_{31}(1910)$, and $P_{33}(1920)$ resonances. For the $P_{11}(1440)$ and the $P_{33}(1600)$ resonances - both described as radial excitations - the present model gives too large masses ${ }^{9}$ as usually happens in a constituent-quark model with spherical symmetry. In particular the Roper resonance is predicted at about $150 \mathrm{MeV}$ higher than the experimental mass. The replacement of the theoretical by the corresponding experimental mass (column "hybrid") does not improve the situation. However, the QPC model gives a stronger $N \pi$ coupling for the $P_{11}(1440)$ than for $P_{11}$ (1710) which is in agreement with the experiment and opposite to the predictions of PSE from Ref. 9.

There are problems for some negative-parity states also. In the calculated spectrum ${ }^{9}$ the $S_{31}(1620)$ is degenerate with the $D_{33}(1700)$ resonance while experimentally the latter lies higher above the first by $\sim 80 \mathrm{MeV}$. The introduction of a spin-orbit coupling can, in principle, lift this degeneracy. This has been proven in Ref. 6. But the spin-orbit coupling does not appear to lift the mentioned degeneracy in the context of the present basis, as shown in Ref. 3 by a variational procedure.

From other studies ${ }^{19}$ there are indications that the inclusion of the $N=3$ band can affect the negative-parity spectrum and in particular the mixing angles of the $S_{11}$ resonances. This might imply a change in the decay widths also.

Besides the deficient description of some baryon resonances by the model used in this work we should also discuss the role of the pion wave function (2.3). As obvious from Eq. (3.5) the pion size is responsible for the amount of nonlocality brought in by the emission operator of the QPC model. A comparison between column 3 (pointlike pion) and column 5 (finite-size pion) helps to draw a pattern of the above-mentioned striking discrepancies. One can see that for the $P_{11}(1440)$ resonance the present finite-size pion gives too large a width but the pointlike pion a too small one. For the resonances $S_{11}(1650)$, $P_{33}(1600), S_{31}(1620)$, and $P_{31}(1910)$ the widths obtained with the finite-size pion are far too small, even taking into account the numerical error due to the Monte Carlo method. On the other hand, the pointlike pion produces larger or much larger values of $\Gamma_{N \pi}$ for these resonances. It seems as if the nonlocality introduced by the pion wave function (2.3) is too large and that a pion of a smaller size, i.e., of a rms radius smaller than $0.29 \mathrm{fm}$ would reduce the striking discrepancy of the theoretical values with respect to the experiment. An exception would remain the $P_{33}(1920)$ resonance for which the width is rather insensitive to the pion size.

\section{DISCUSSION}

We have calculated the pion decay widths of 19 nonstrange resonances by using the quark-pair-creation model ${ }^{3} P_{0}$. The baryon and the pion were treated as particles having a structure derived from the same QCDmotivated flux-tube model. The quark-pair-creation model can be related to a flux-tube-breaking mechanism. Here we analyzed the case where the flux tube has an infinite extension.

We found that with only one free parameter the quark-pair-creation model gives results comparable to those obtained from the pseudoscalar-emission model with two free parameters and much better than those obtained from the one-parameter PSE model. Therefore, it seems important to treat the pion as a finite-size particle, described on an equal footing with the baryon by a QCD-inspired model. It would also be interesting to study whether a breaking mechanism based on a finite extension flux tube can influence the decay widths.

The ${ }^{3} P_{0}$ model also proved successful in other studies as, e.g., the $p \bar{p}$ or $\Lambda \bar{\Lambda}$ annihilation into two or three mesons. ${ }^{20,21}$ Further application of the ${ }^{3} P_{0}$ model to the analysis of strong decay of strange baryons is therefore desirable. 


\section{ACKNOWLEDGMENTS}

We would like to thank Jack Paton for several useful discussions and Renato Sartor for good advice and help in the numerical integration with the Monte Carlo method. One of us (F.S.) acknowledges the kind hospitality of the Theory Group at TRIUMF where part of this work has been done.

\section{APPENDIX}

The nine-dimensional integral (3.5) has been calculated numerically using a Monte Carlo procedure. We used spherical coordinates for the factors $\rho, \lambda$, and $\mathbf{x}$ and re- placed $\rho, \lambda$, and $x$ by new variables $\alpha_{i}(i=1,2,3)$ given by the transformation

$$
\rho=\tan \alpha_{1}, \quad \lambda=\tan \alpha_{2}, \quad x=\tan \alpha_{3} .
$$

This transformation helps to increase the numerical accuracy by smoothing the integrand.

The integration upper limits of the variables given by (A1) have been set equal to $\tan (\pi / 3)$. The program has been run over 50000 random points so that the numerical error estimated on a standard deviation basis has been kept below $20 \%$ for most of the resonances. For $P_{33}(1600), P_{33}(1920)$, and $P_{11}(1710)$ we could not reach a good numerical precision.
${ }^{1}$ J. Carlson, J. Kogut, and V. R. Pandharipande, Phys. Rev. D 27, 23 (1983).

${ }^{2}$ N. Isgur and J. Paton, Phys. Rev. D 31, 2910 (1985).

${ }^{3}$ J. Carlson, J. Kogut, and V. R. Pandharipande, Phys. Rev. D 28, 2807 (1983).

${ }^{4}$ R. Kokoski and N. Isgur, Phys. Rev. D 35, 907 (1987).

${ }^{5}$ R. Sartor and Fl. Stancu, Phys. Rev. D 31, 128 (1985).

${ }^{6}$ S. Capstick and N. Isgur, Phys. Rev. D 34, 2809 (1986).

${ }^{7}$ L. Micu, Nucl. Phys. B10, 521 (1969); R. Carlitz and M. Kislinger, Phys. Rev. D 2, 336 (1970).

${ }^{8}$ A. Le Yaouanc, L. Oliver, O. Pène, and J.-C. Raynal, Phys. Rev. D 8, 2223 (1973); 9, 1415 (1974); 11, 1272 (1975).

${ }^{9}$ R. Sartor and Fl. Stancu, Phys. Rev. D 34, 3405 (1986).

${ }^{10}$ A. N. Mitra and M. Ross, Phys. Rev. 158, 1630 (1967).

${ }^{11}$ R. Koniuk and N. Isgur, Phys. Rev. D 21, 1868 (1980).

${ }^{12}$ A. de Rujula, H. Georgi, and S. L. Glashow, Phys. Rev. D 12, 147 (1975).

${ }^{13}$ R. Sartor and Fl. Stancu (unpublished).
${ }^{14}$ Particle Data Group, M. Aguilar-Benitez et al., Phys. Lett. 170B, 1 (1986).

${ }^{15}$ G. Hoeler, F. Kaiser, R. Koch, and E. Pietarinen, Handbook of Pion-Nucleon Scattering (Physics Data, No. 12-1) (Fachsinformationzentrum, Karlsruhe, 1979).

${ }^{16}$ R. E. Cutkosky, C. P. Forsyth, J. P. Babcock, R. L. Kelly, and R. E. Hendrick, in Baryon 1980, proceedings of the IVth International Conference on Baryon Resonances, Toronto, edited by N. Isgur (University of Toronto, Toronto, 1981), p. 19.

${ }^{17}$ M. B. Gavela, A. Le Yaouanc, L. Oliver, O. Pène, J. C. Raynal, and S. Sood, Phys. Rev. D 21, 182 (1980).

${ }^{18}$ R. Sartor and Fl. Stancu, Phys. Rev. D 33, 727 (1986).

${ }^{19}$ C. P. Forsyth and R. E. Cutkosky, Z. Phys. C 18, 219 (1983).

${ }^{20}$ M. J. Soulliere, Ph.D. thesis, Pennsylvania State University, 1987, for the experimental situation and a review of the theoretical work.

${ }^{21}$ S. Furui, A. Faessler, and S. Ono, University of Tübingen report, 1987 (unpublished). 